\title{
RHBDD1 promotes proliferation, migration, invasion and EMT in renal cell carcinoma via the EGFR/AKT signaling pathway
}

\author{
MINGYANG LI ${ }^{1,2}$, LICHENG CAI ${ }^{1}$, XINGYUAN WANG ${ }^{1}$, YIPENG YU ${ }^{1}$, WENGANG JIAN ${ }^{1}$, GUOCHANG BAO $^{2}$, \\ ZHIMING GAO ${ }^{2}$, JUNSHENG GUO ${ }^{2}$, JIAN ZHANG ${ }^{2},{\text { CHUNSHENG } \text { LI }^{2} \text { and CHENG ZHANG }}^{1}$ \\ ${ }^{1}$ Department of Urology, First Affiliated Hospital of Harbin Medical University, Harbin, Heilongjiang 150001; \\ ${ }^{2}$ Department of Urology, Affiliated Hospital of Chifeng University, Chifeng, \\ Inner Mongolia Autonomous Region 024005, P.R. China
}

Received June 29, 2020; Accepted January 12, 2021

DOI: $10.3892 / \mathrm{mmr} .2021 .12466$

\begin{abstract}
Renal cell carcinoma (RCC) is a common malignant tumor of the urinary system with a poor prognosis and high mortality rate. The increasing incidence of RCC poses a serious threat to human health. It is well-documented that rhomboid domain-containing protein 1 (RHBDD1) plays a vital role in cancer progression. The present study was designed to identify the biological functions of RHBDD1 in RCC and investigate the underlying regulatory mechanism, aiming to explore the novel molecular therapeutic targets for RCC. The protein and mRNA expression levels of RHBDD1 in normal renal tubule epithelium and human RCC cell lines were analyzed using western blotting and reverse transcription-quantitative PCR. Cell proliferation was determined using Cell Counting Kit- 8 assays. Wound healing and Transwell assays were performed to determine cell migration and invasion, respectively. In addition, key proteins related to migration, invasion and epithelial-mesenchymal transition (EMT), such as matrix metalloproteinase (MMP)2, MMP9, MMP13, E-cadherin, $\mathrm{N}$-cadherin, vimentin and Slug, were analyzed using western blotting. In addition, the EGFR/AKT signaling pathway was further studied using western blotting to determine the potential molecular mechanism. The results of the present study revealed that RHBDD1 expression levels were significantly upregulated in RCC cell lines. The knockdown of RHBDD1 inhibited cell proliferation, migration, invasion and EMT,
\end{abstract}

Correspondence to: Dr Cheng Zhang, Department of Urology, First Affiliated Hospital of Harbin Medical University, 23 Post Road, Nangang, Harbin, Heilongjiang 150001, P.R. China

E-mail: chengzhang4321@yeah.net

Mr. Chunsheng Li, Department of Urology, Affiliated Hospital of Chifeng University, 42 Wangfu Street, New Town, Songshan, Chifeng, Inner Mongolia Autonomous Region 024005, P.R. China E-mail: chunshengli123@yeah.net

Key words: rhomboid domain-containing protein 1, proliferation, migration, invasion, epithelial-mesenchymal transition, renal cell carcinoma, EGFR/AKT while the overexpression of RHBDD1 promoted cell proliferation, migration, invasion and EMT in RCC. In addition, the knockdown of RHBDD1 suppressed the activation of the EGFR/AKT signaling pathway, while the overexpression of RHBDD1 activated the EGFR/AKT signaling pathway. Moreover, these stimulatory effects of RHBDD1 overexpression on RCC progression and the EGFR/AKT signaling pathway were partly reversed by gefitinib, an EGFR inhibitor. In conclusion, the findings of the present study suggested that RHBDD1 may be a crucial regulator of RCC by modulating the EGFR/AKT signaling pathway. The present study may provide a theoretical basis and potential targets for RCC treatment.

\section{Introduction}

Renal cell carcinoma ( RCC) is one of the most common types of malignant cancer of the urinary system, accounting for $2-3 \%$ of adult malignant tumors (1). The early diagnosis of $\mathrm{RCC}$ is difficult and the risk of cancer metastasis is high due to the undetermined mechanism of onset of the disease and non-specific clinical manifestations (2). Metastatic RCC has a poor prognosis with high recurrent rate and mortality and accounts for $20-30 \%$ of newly diagnosed RCC cases (3). It is not sensitive to radiotherapy or chemotherapy, and traditional cytokine therapy has limited efficacy in patients with metastatic RCC (4,5). In recent years, following the emergence of targeted drugs and immune checkpoint inhibitors, the overall survival and progression-free survival of patients with metastatic RCC have greatly improved (6).

Tumor development is a complex multifactorial process. Matrix metalloproteinases (MMPs) are key enzymes involved in the process of cancer invasion and metastasis (7). MMPs have been reported to degrade the extracellular matrix and basement membrane, facilitate the formation of vessels and enhance cell invasion and metastasis in $\operatorname{RCC}(8,9)$. Epithelial-mesenchymal transition (EMT) is a process in which epithelial cells transform into mesenchymal cells (10). Previous studies have demonstrated that EMT could regulate numerous genes (such as Twist, Slug, Snail and zinc finger E-box-binding homeobox 1) and signaling pathways (such as Wnt/ $\beta$-catenin, Notch/Jagged and Hedgehog signaling pathway) to influence 
the invasion and metastasis of tumors $(11,12)$. Thus, regulating the EMT process and the expression levels of MMPs may represent a key axis for RCC prevention and therapy (13).

EGFR is a member of the tyrosine kinase type I receptor family and a well-established target for cancer therapy (14). EGFR inhibitors have been approved for the treatment of multiple types of cancer, such as non-small cell lung cancer, colon cancer and glioblastoma (15). AKT is an important member of the PI3K signaling pathway (16). Thus, determining the effect of AKT inhibitors may represent a novel targeted drug treatment for cancer as $>50 \%$ of tumors are overactivated by AKT $(17,18)$. A previous study reported that inhibiting the activity of the EGFR/AKT signaling pathway regulated RCC growth, metastasis and EMT (19). Thus, it is of urgent significance to identify effective methods to suppress the EGFR/AKT signaling pathway.

Rhomboid domain-containing protein 1 (RHBDD1) is a serine protease that has a role in regulated intramembrane proteolysis (20). RHBDD1 has been identified to serve a role in cancer. For example, RHBDD1 expression levels were upregulated in breast cancer (21). In vitro, knockdown of RHBDD1 could inhibit cell proliferation, migration, invasion and EMT in breast cancer (22). Moreover, Song et al (23) discovered that RHBDD1 could promote colorectal cancer growth by activating the EGFR signaling pathway.

The present study aimed to investigate the functional role of RHBDD1 in RCC, in addition to its underlying molecular mechanism. In more detail, the study investigated the influence of RHBDD1 on RCC cell proliferation, migration, invasion and EMT in vitro and sought to determine whether RHBDD1 exerted its functions through modulating the EGFR/AKT signaling pathway.

\section{Materials and methods}

Cell culture. Normal renal tubule epithelium cell lines, HKC 5 and HK-2, and human RCC cell lines, 7860 and Caki-1, were purchased from The Cell Bank of Type Culture Collection of The Chinese Academy of Sciences. The human RCC cell lines, LoMet-ccRCC and A498, were obtained from the China Infrastructure of Cell Line Resources, Institute of Basic Medical Sciences, Chinese Academy of Medical Sciences. Cells were cultured in DMEM (Gibco; Thermo Fisher Scientific, Inc.) supplemented with 10\% FBS (Gibco; Thermo Fisher Scientific, Inc.), $100 \mathrm{U} / \mathrm{ml}$ penicillin and $100 \mathrm{mg} / \mathrm{ml}$ streptomycin (Invitrogen; Thermo Fisher Scientific, Inc.), and maintained in an atmosphere containing $5 \% \mathrm{CO}_{2}$ and $95 \%$ air at $37^{\circ} \mathrm{C}$.

Cell transfection. The pGPU6/GFP/Neo vector (Shanghai GenePharma Co., Ltd.) was used to construct a vector containing short hairpin RNA (shRNA) targeting RHBDD1. A RHBDD1 genomic fragment was cloned into a pcDNA3.1 vector (Shanghai GenePharma Co., Ltd.) to overexpress RHBDD1. The empty vectors served as their negative controls (NCs), shRNA-NC or OV-NC, respectively. For transfection, cells were plated into six-well plates and cultured to $90 \%$ confluence. Subsequently, $50 \mathrm{nM}$ individual vectors [shRNA-NC, shRNA-RHBDD1-1, shRNA-RHBDD1-2, overexpression (Ov)-NC or Ov-RHBDD1] and $5 \mu 1$ Lipofectamine ${ }^{\circledR} 2000$ reagent (Invitrogen; Thermo Fisher Scientific, Inc.) were separately incubated in serum-free Opti-MEM (Gibco; Thermo Fisher Scientific, Inc.) for $5 \mathrm{~min}$ at room temperature and then mixed for another $20 \mathrm{~min}$ incubation at room temperature. Subsequently, the transfection mixture was added into cells and cultured for $6-8 \mathrm{~h}$ at $37^{\circ} \mathrm{C}$. Following the incubation, the medium was replaced with complete DMEM and cultured at $37^{\circ} \mathrm{C}$ for $48 \mathrm{~h}$ for use in subsequent experiments.

Cell treatment. Cells transfected with Ov-RHBDD1 were treated with $1 \mu \mathrm{M}$ gefitinib (Selleck Chemicals) in the presence of $10 \% \mathrm{FBS}$ at $37^{\circ} \mathrm{C}$ for $24 \mathrm{~h}$.

Reverse transcription-quantitative PCR (RT- $q P C R)$. Total RNA was extracted from cells using RNA-Trip reagent (Applygen Technologies, Inc.). Total RNA was reverse transcribed into cDNA using a PrimeScript RT reagent kit with gDNA Eraser (Takara Bio, Inc.) according to the manufacturer's instructions. qPCR was subsequently performed using SYBR Green PCR reagent kit (Applied Biosystems; Thermo Fisher Scientific, Inc.) on an ABI 7000 Real-Time PCR Detection system (Applied Biosystems; Thermo Fisher Scientific, Inc.). The following thermocycling conditions were used for the qPCR: Initial denaturation at $95^{\circ} \mathrm{C}$ for $5 \mathrm{~min}$; followed by 40 cycles of denaturation at $95^{\circ} \mathrm{C}$ for $10 \mathrm{sec}$, annealing at $60^{\circ} \mathrm{C}$ for $30 \mathrm{sec}$ and extension at $72^{\circ} \mathrm{C}$ for $35 \mathrm{sec}$, and a final extension at $72^{\circ} \mathrm{C}$ for $5 \mathrm{~min}$. The following primer pairs were used for the qPCR: RHBDD1 forward, 5'-ATCTG GCTGGGATTCTTGTTG-3' and reverse, 5'-GGCTGGCTTG TAATGCTCTC-3'; and GAPDH forward, 5'-TCAACGACC ACTTTGTCAAGCTCA-3' and reverse, 5'-GCTGGTGGTCC AGGGGTCTTACT-3'. The relative expression levels were quantified using the $2^{-\Delta \Delta C q}$ method (24) and RHBDD1 expression levels were normalized to GAPDH.

Nuclear/cytoplasmic separation. A Nuclear Protein Extraction kit (Beijing Solarbio Science \& Technology Co., Ltd.) was used to separate the cytoplasmic and nuclear proteins, according to the manufacturer's protocol. The expression levels of Ki67 and proliferating cell nuclear antigen (PCNA) in nuclear proteins were detected by western blotting.

Western blotting. Total protein was extracted from cells using RIPA lysis buffer (Beyotime Institute of Biotechnology) supplemented with proteinase inhibitors. Following centrifugation at $12,000 \mathrm{x}$ g for $10 \mathrm{~min}$ at $4^{\circ} \mathrm{C}$, protein quantification was performed using a BCA Protein Assay kit (Beyotime Institute of Biotechnology) and equal amounts of protein (30 $\mu \mathrm{g} /$ lane) were separated via SDS-PAGE on $8-10 \%$ gels. The proteins were subsequently transferred onto PVDF membranes (Beyotime Institute of Biotechnology) and blocked in 5\% BSA (Beijing Solarbio Science \& Technology Co., Ltd.) for $1 \mathrm{~h}$ at room temperature. The membranes were then incubated with the following primary antibodies at $4^{\circ} \mathrm{C}$ overnight: Anti-RHBDD1 (Abcam; cat. no. ab254805; 1:1,000; $34 \mathrm{kDa}$ ), anti-Ki67 (Abcam; cat. no. ab16667; 1:1,000; $358 \mathrm{kDa}$ ), anti-PCNA (Abcam; cat. no. ab18197; 1:1,000; $29 \mathrm{kDa}$ ), anti-MMP2 (Abcam; cat. no. ab97779; 1:3,000; $74 \mathrm{kDa}$.), anti-MMP9 (Abcam; cat. no. ab228402; 1:1,000; $81 \mathrm{kDa}$ ), anti-MMP13 (Abcam; cat. no. ab51072; 1:1,000; $54 \mathrm{kDa}$ ), anti-E-cadherin (Abcam; cat. no. ab40772; 
A

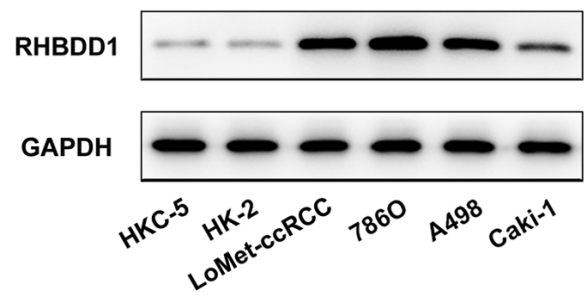

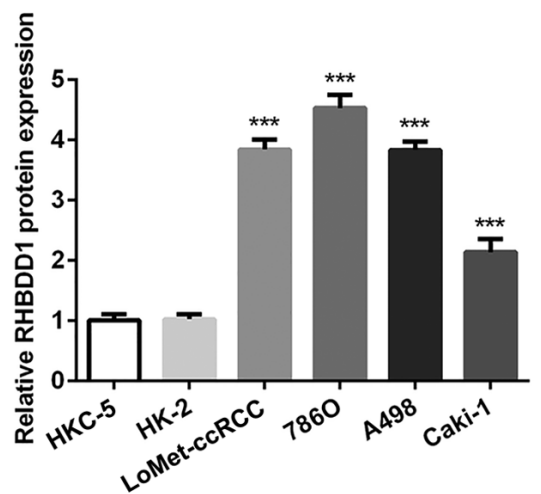

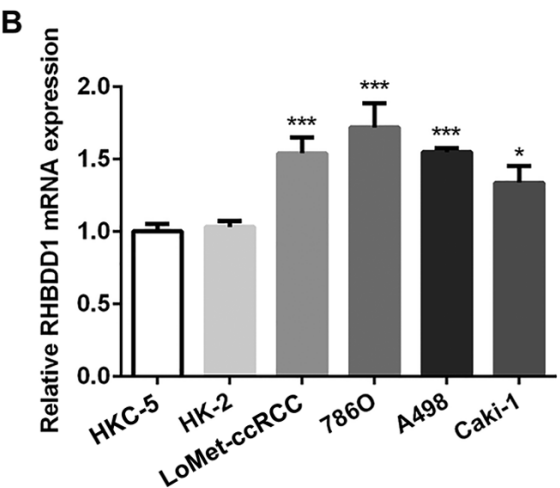

Figure 1. RHBDD1 expression levels are significantly upregulated in RCC cell lines. (A) Protein and (B) mRNA expression levels of RHBDD1 in normal renal tubule epithelium cell lines, HKC-5 and HK-2, and human RCC cell lines, 786O, LoMet-ccRCC, A498 and Caki-1, were determined using western blotting and reverse transcription-quantitative PCR, respectively. ${ }^{*} \mathrm{P}<0.05,{ }^{* * * *} \mathrm{P}<0.001$ vs. HKC-5 and HK-2 cell lines. RHBDD1, rhomboid domain-containing protein 1 ; RCC, renal cell carcinoma.

1:10,000; $97 \mathrm{kDa})$, anti-N-cadherin (Abcam; cat. no. ab18203; 1:1,000; $100 \mathrm{kDa}$ ), anti-vimentin (Abcam; cat. no. ab137321; 1:3,000; $54 \mathrm{kDa}$ ), anti-Slug (Abcam; cat. no. ab27568; 1:1,000; $30 \mathrm{kDa}$ ), anti-EGFR (Abcam; cat. no. ab52894; 1:10,000; $134 \mathrm{kDa})$, anti-phosphorylated (p)-AKT (Abcam; cat. no. ab38449; 1:1,000; $56 \mathrm{kDa}$ ), anti-AKT (Abcam; cat. no. ab8805; 1:500; $56 \mathrm{kDa}$ ), anti-Lamin B1 (Abcam; cat. no. ab133741; 1:10,000; $66 \mathrm{kDa})$ and anti-GAPDH (Abcam; cat. no. ab8245; 1:10,000; $40.2 \mathrm{kDa}$ ). Following the primary antibody incubation, the membranes were incubated with a goat anti-rabbit HRP-conjugated secondary antibody (Abcam; cat. no. ab205718; 1:50,000) or a goat anti-mouse HRP-conjugated secondary antibody (Abcam; cat. no. ab205719; 1:20,000) at room temperature for $1 \mathrm{~h}$. Protein bands were visualized using an ImageQuant LAS 4000 apparatus (Cytiva) and analyzed using ImageJ software (version 1.47; National Institutes of Health).

Cell Counting Kit-8 (CCK-8) assay. Cell viability was analyzed using a CCK-8 assay (Beyotime Institute of Biotechnology) according to the manufacturer's protocol. Briefly, cells were seeded at a density of $5 \times 10^{4}$ cells $/ \mathrm{ml}$ in an incubator with standard culture conditions $\left(37^{\circ} \mathrm{C}, 5 \% \mathrm{CO}_{2}\right)$ for $24 \mathrm{~h}$ following the aforementioned transfections and gefitinib treatment. Following the incubation, $10 \mu \mathrm{l} /$ well CCK- 8 solution was added and incubated at $37^{\circ} \mathrm{C}$ for another $4 \mathrm{~h}$. Finally, the absorbance was measured at a wavelength of $450 \mathrm{~nm}$ used a microplate reader (Thermo Fisher Scientific, Inc.) to obtain the optical density value.

Wound healing assay. Following the aforementioned transfections and gefitinib treatment, cells were cultured in DMEM supplemented with 10\% FBS for use in the wound healing assay. Upon reaching $90 \%$ confluence, the cell monolayer was scratched with a $200-\mu l$ pipette tip and the detached cells were washed with PBS. Subsequently, the cells were cultured at $37^{\circ} \mathrm{C}$ in serum-free DMEM. Cells migrating into the wound sites were analyzed over a $24 \mathrm{~h}$ period, with plates being imaged at 0 and $24 \mathrm{~h}$ under an inverted light microscope (magnification, x100; Leica Microsystems GmbH). Cell migration was quantified by determining the extent (\%) of wound healing as follows: ( $0 \mathrm{~h}$ scratch area $-24 \mathrm{~h}$ scratch area) / scratch area at $0 \mathrm{~h} \times 100 \%$.
Transwell invasion assay. The invasive ability of cells was analyzed using Transwell chambers precoated with Matrigel (Beijing Solarbio Science \& Technology Co., Ltd.) for $8 \mathrm{~h}$ at $37^{\circ} \mathrm{C}$. Briefly, $2 \times 10^{5}$ cells $/ \mathrm{ml}$ suspended in serum-free DMEM were plated into the upper chambers of Transwell plates, while DMEM supplemented with $10 \%$ FBS as a chemoattractant was plated into the lower chambers. Following incubation for $24 \mathrm{~h}$ at $37^{\circ} \mathrm{C}$, non-invasive cells were removed and the invasive cells in the lower chambers of the Transwell plates were fixed with $4 \%$ paraformaldehyde for $30 \mathrm{~min}$ and stained with $0.4 \%$ crystal violet (Sigma-Aldrich; Merck KGaA) for $5 \mathrm{~min}$ at room temperature. Invasive cells were counted in five randomly selected fields of view using an inverted light microscope (magnification, x100; Leica Microsystems GmbH).

Statistical analysis. Experiments were performed in triplicate and data are presented as mean \pm standard deviation. Statistical differences among groups were determined using one-way ANOVA followed by a Tukey's post hoc test. Statistical analysis was performed using GraphPad Prism 6.01 software (GraphPad Software, Inc.). P $<0.05$ was considered to indicate a statistically significant difference.

\section{Results}

RHBDD1 expression levels are upregulated in RCC cell lines. To determine whether RHBDD1 was involved in RCC, RHBDD1 expression levels were analyzed in the normal renal tubule epithelium cell lines, HKC-5 and HK-2, and the human RCC lines, 786O, LoMet-ccRCC, A498 and Caki-1, using western blotting and RT-qPCR. Compared with normal renal tubule epithelium cell lines, the protein (Fig. 1A) and mRNA (Fig. 1B) expression levels of RHBDD1 were significantly upregulated in RCC cell lines. These findings suggested that RHBDD1 may play an important role in RCC. The expression levels of RHBDD1 were the highest in the RCC cell line, 7860. Therefore, 7860 cells were selected for use in subsequent experiments.

Knockdown of RHBDDI inhibits cell proliferation in RCC. As RHBDD1 expression may be associated with the progression of RCC, shRNA-RHBDD1-1 and shRNA-RHBDD1-2 

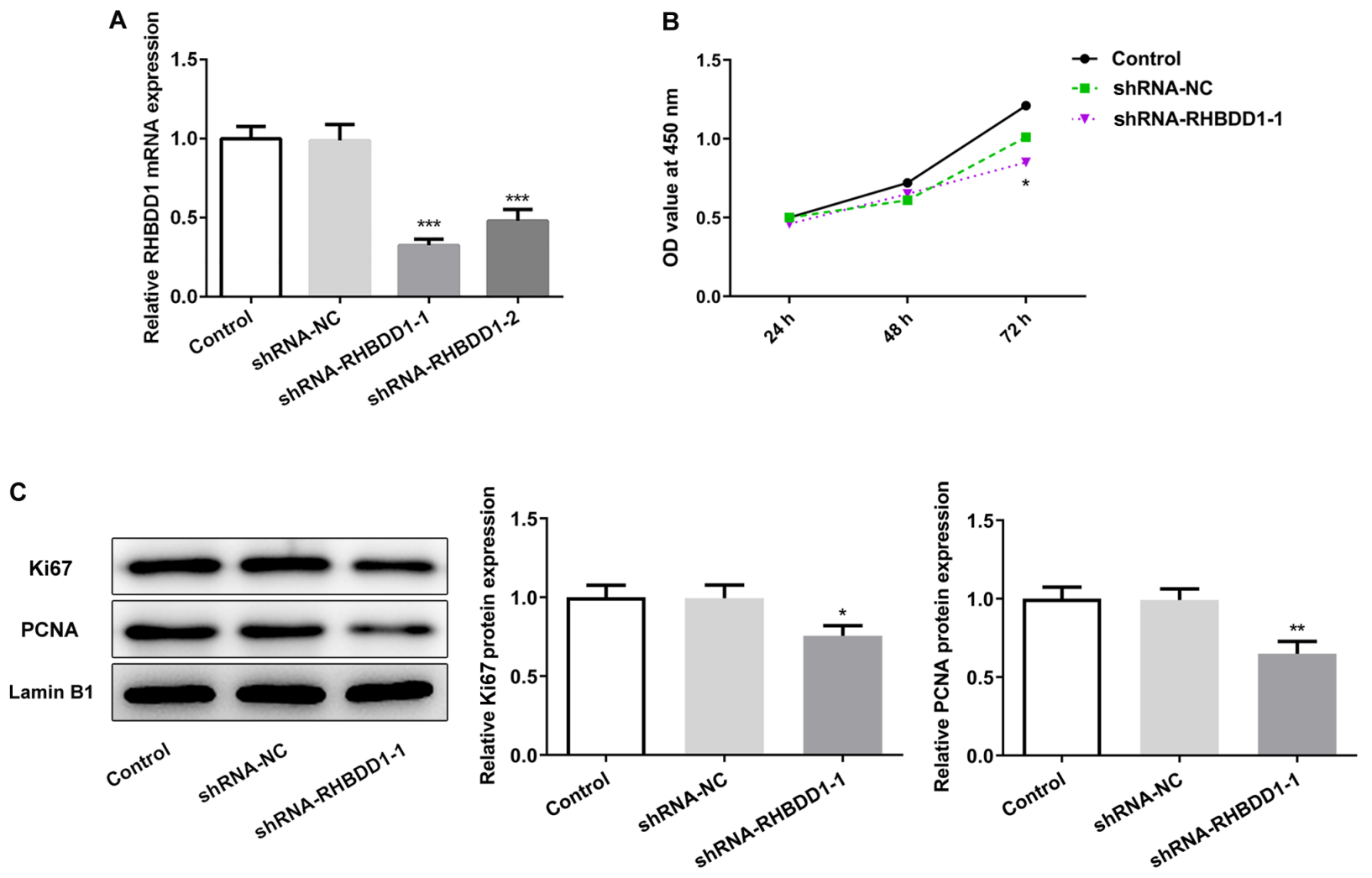

Figure 2. Knockdown of RHBDD1 inhibits cell proliferation in renal cell carcinoma. (A) RHBDD1 expression levels in $786 \mathrm{O}$ cells were analyzed using reverse transcription-quantitative PCR. (B) Cell proliferation was assessed using a Cell Counting Kit-8 assay. (C) Western blotting was used to analyze the expression levels of Ki67 and PCNA. "P $<0.05,{ }^{* *} \mathrm{P}<0.01,{ }^{* * *} \mathrm{P}<0.001$ vs. shRNA-NC. RHBDD1, rhomboid domain-containing protein 1; PCNA, proliferating cell nuclear antigen; OD, optical density; shRNA, short hairpin RNA; NC, negative control.

were transfected into the 7860 cell line to determine the biological functions of RHBDD1 in RCC. Compared with the shRNA-NC group, transfection with shRNA-RHBDD1 significantly downregulated the expression levels of RHBDD1. Due to the optimized transfection efficiency, shRNA-RHBDD1-1 was chosen for the functional experiments (Fig. 2A). Data obtained from CCK-8 assay revealed that cell proliferation was significantly inhibited following RHBDD1 knockdown in comparison with the shRNA-NC group (Fig. 2B). Moreover, the expression levels of the proliferation-associated proteins, Ki67 and PCNA, were analyzed using western blotting to further verify the effects of RHBDD1 on cell proliferation in vitro. As expected, the protein expression levels of Ki67 and PCNA were significantly downregulated in the $786 \mathrm{O}$ cell lines following the transfection with shRNA-RHBDD1-1 compared with the shRNA-NC group (Fig. 2C). These results suggested that the knockdown of RHBDD1 expression may suppress RCC cell proliferation.

Knockdown of RHBDD1 suppresses cell migration, invasion and EMT in RCC. The present study subsequently aimed to determine the function of RHBDD1 in cell migration, invasion and EMT of RCC. The results of the wound healing assay revealed that the migration of 7860 cells was significantly reduced following the transfection with shRNA-RHBDD1-1 compared with the shRNA-NC group (Fig. 3A). Similarly, the knockdown of RHBDD1 significantly decreased RCC cell invasion compared with the shRNA-NC group (Fig. 3B). In addition, western blotting was performed to analyze the expression levels of key proteins related to cell migration and invasion. MMP2, MMP9 and MMP13 expression levels were significantly downregulated following the knockdown of RHBDD1 expression compared with the shRNA-NC group, which indicated that the knockdown of RHBDD1 may suppress cell migration and invasion in RCC (Fig. 3C). The expression levels of several EMT markers, including E-cadherin, $\mathrm{N}$-cadherin, vimentin and Slug, were also analyzed to determine the underlying mechanism of RHBDD1 in tumor progression. The expression levels of E-cadherin were upregulated, while the expression levels of $\mathrm{N}$-cadherin, vimentin and Slug were downregulated following the knockdown of RHBDD1 compared with the shRNA-NC group, which suggested that RHBDD1 may suppress EMT in RCC (Fig. 3D).

Knockdown of RHBDDI inhibits EGFR/AKT signaling pathway in $R C C$. To determine the molecular mechanism through which RHBDD1 may regulate RCC, the expression levels of EGFR/AKT signaling pathway-related proteins were analyzed using western blotting. EGFR and p-AKT expression levels were significantly downregulated following the transfection with shRNA-RHBDD1-1 compared with the shRNA-NC group, indicating that RHBDD1 silencing may suppress EGFR/AKT signaling pathway (Fig. 4A and B).

RHBDD1 promotes cell proliferation via EGFR/AKT signaling pathway in RCC. To further investigate the underlying molecular mechanism through which RHBDD1 regulated cell proliferation in RCC, RHBDD1 overexpression vectors were 
ShRNA-NC
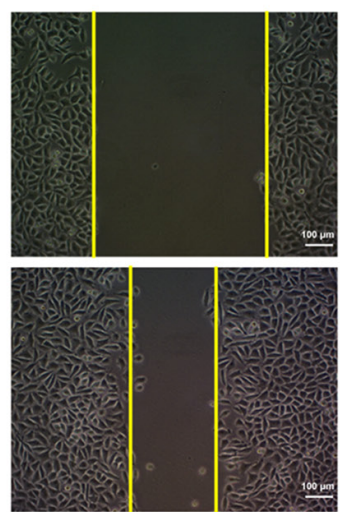

shRNA-NC

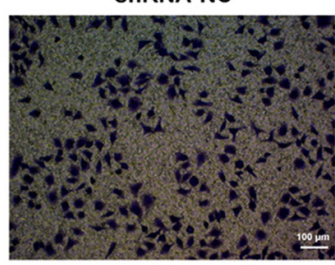

ShRNA-RHBDD1-1
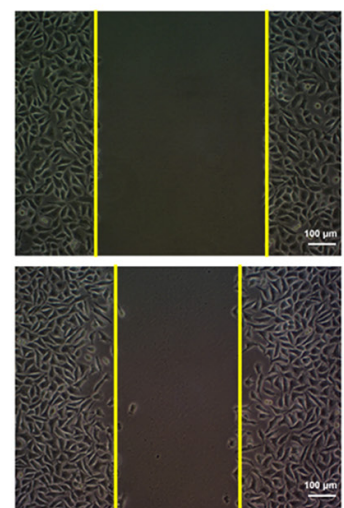

ShRNA-RHBDD1-1

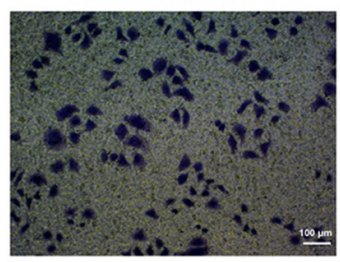

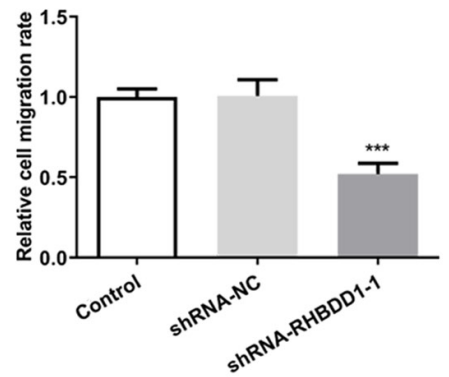
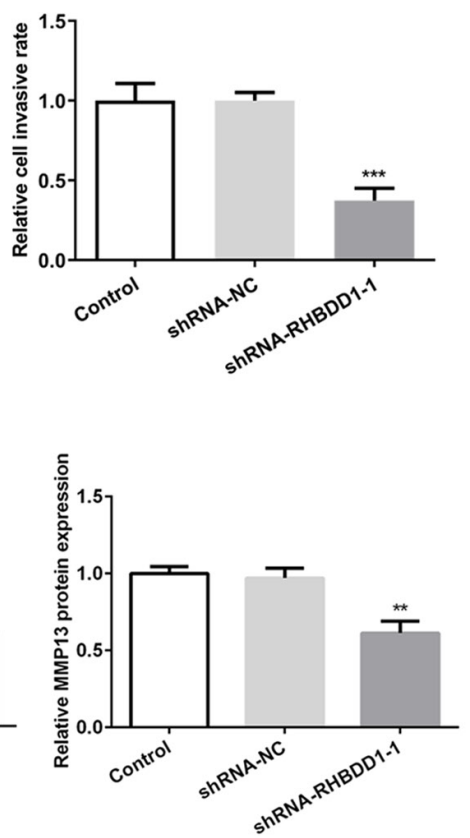
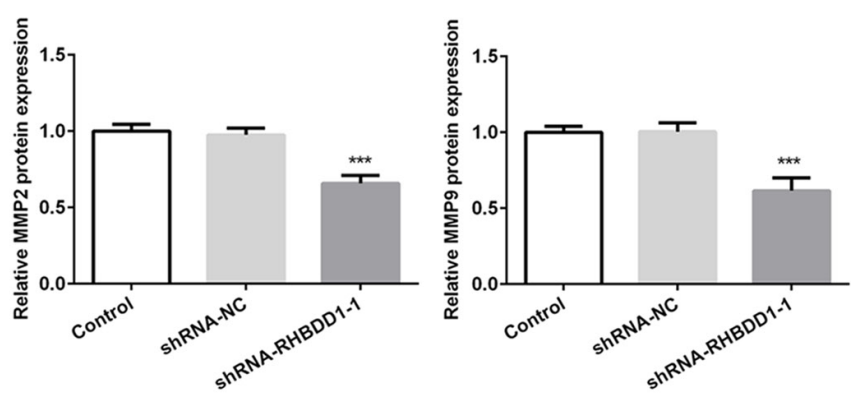

A
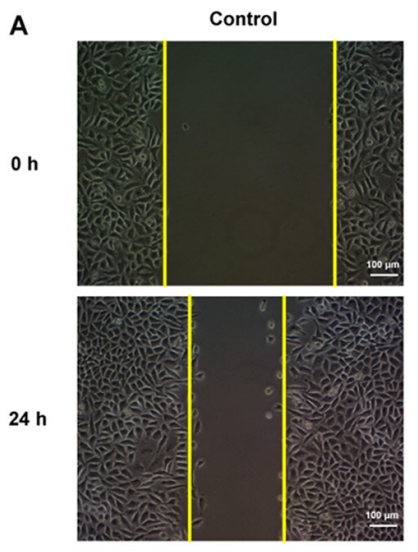

B

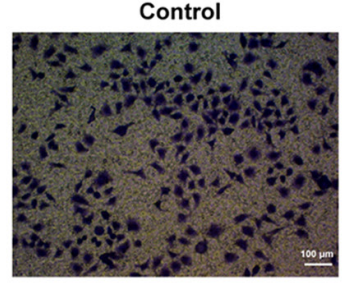

D

C
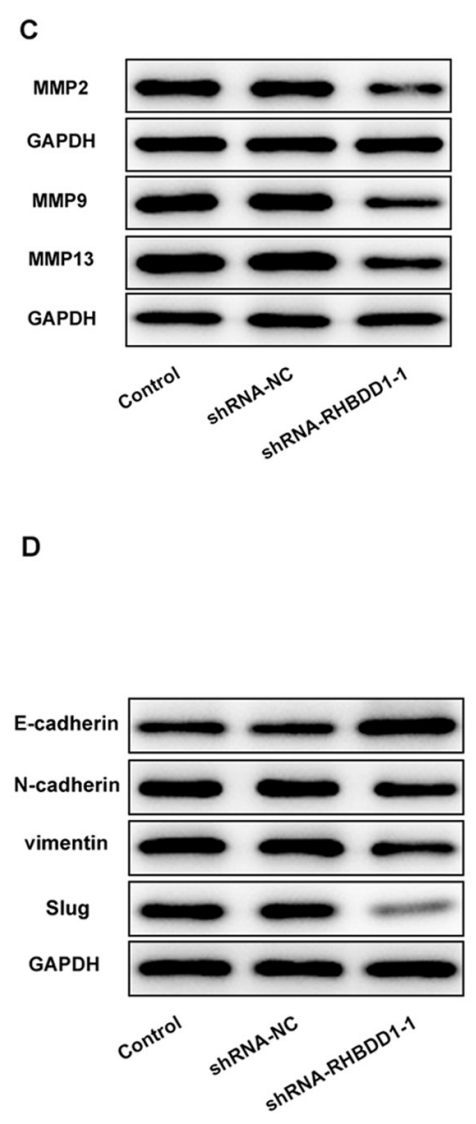
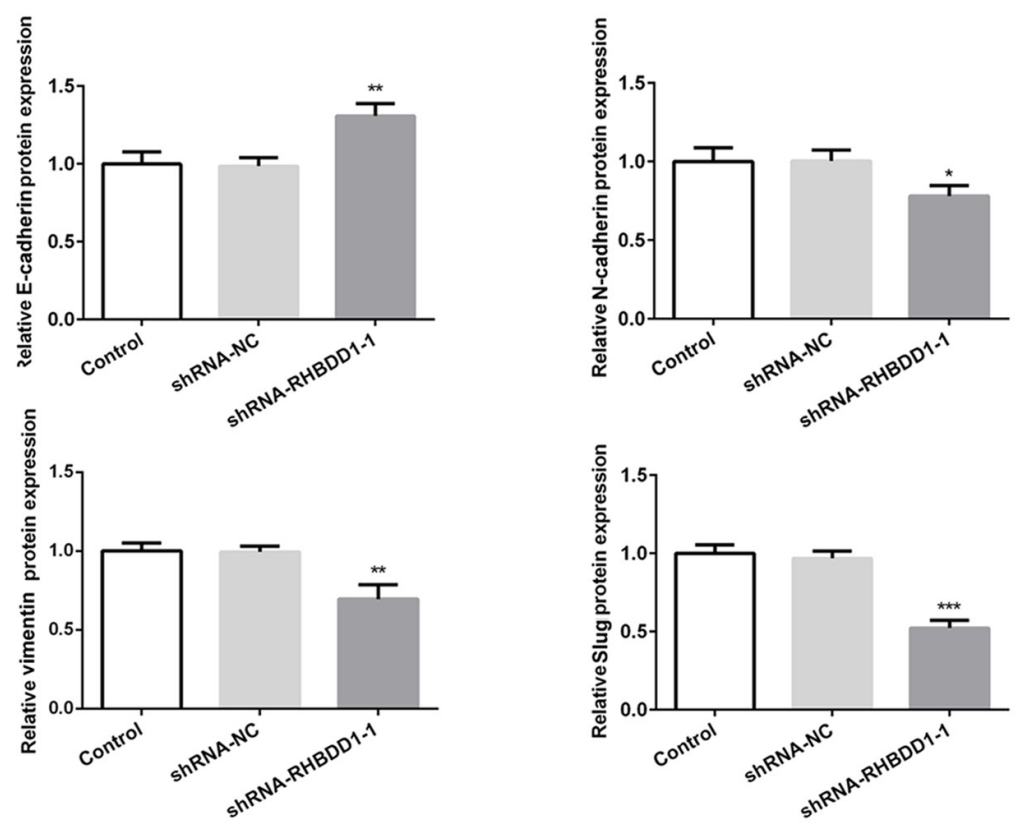

Figure 3. Knockdown of RHBDD1 suppresses cell migration, invasion and EMT in renal cell carcinoma. (A) Wound healing assay was performed to determine cell migration (magnification, x100; scale bar, $100 \mu \mathrm{m}$ ). (B) Transwell assay was performed to determine cell invasion (magnification, x100; scale bar, $100 \mu \mathrm{m}$ ). Western blotting was performed to analyze the expression levels of proteins involved in (C) cell migration and invasion, including MMP2, MMP9 and MMP13 and (D) EMT, including E-cadherin, N-cadherin, vimentin and Slug. ${ }^{*} \mathrm{P}<0.05,{ }^{* * *} \mathrm{P}<0.01,{ }^{* * *} \mathrm{P}<0.001$ vs. shRNA-NC. RHBDD1, rhomboid domain-containing protein 1; EMT, epithelial-mesenchymal transition; MMP, matrix metalloproteinase; shRNA, short hairpin RNA; NC, negative control. 

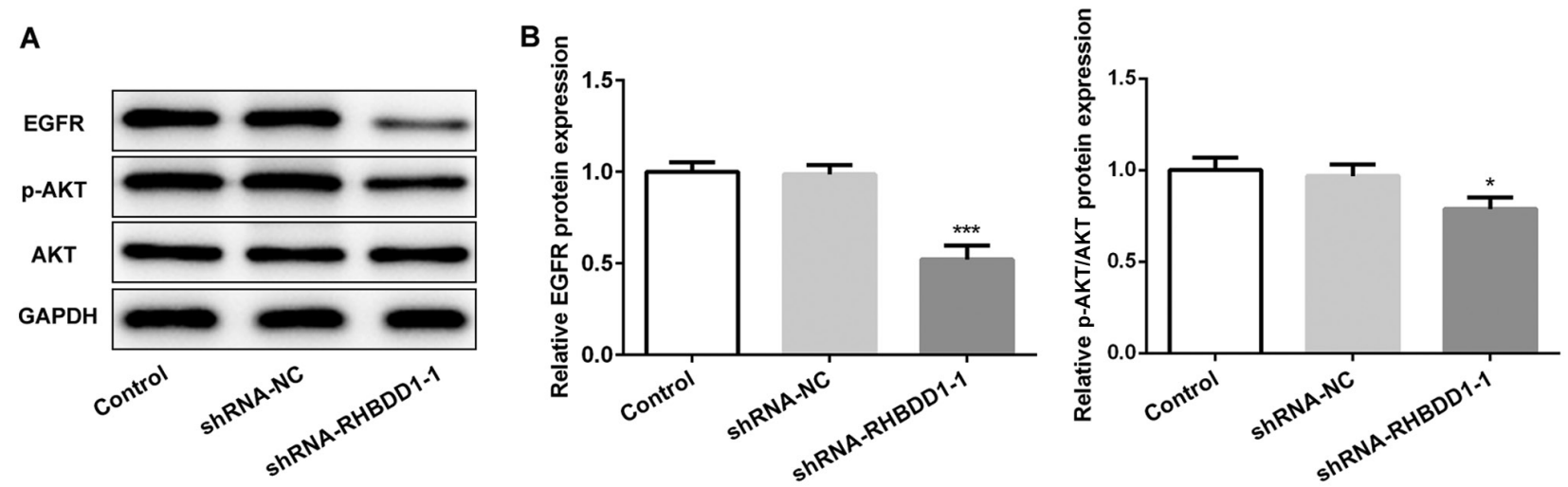

Figure 4. Knockdown of RHBDD1 inhibits EGFR/AKT signaling pathway in renal cell carcinoma. (A) Western blotting was used to analyze EGFR, p-AKT and AKT expression levels. (B) Expression levels of EGFR, p-AKT and AKT from part (A) were semi-quantified. ${ }^{*} \mathrm{P}<0.05$, ${ }^{* * *} \mathrm{P}<0.001 \mathrm{vs}$. shRNA-NC. RHBDD1, rhomboid domain-containing protein 1; p-, phosphorylated; shRNA, short hairpin RNA; NC, negative control.
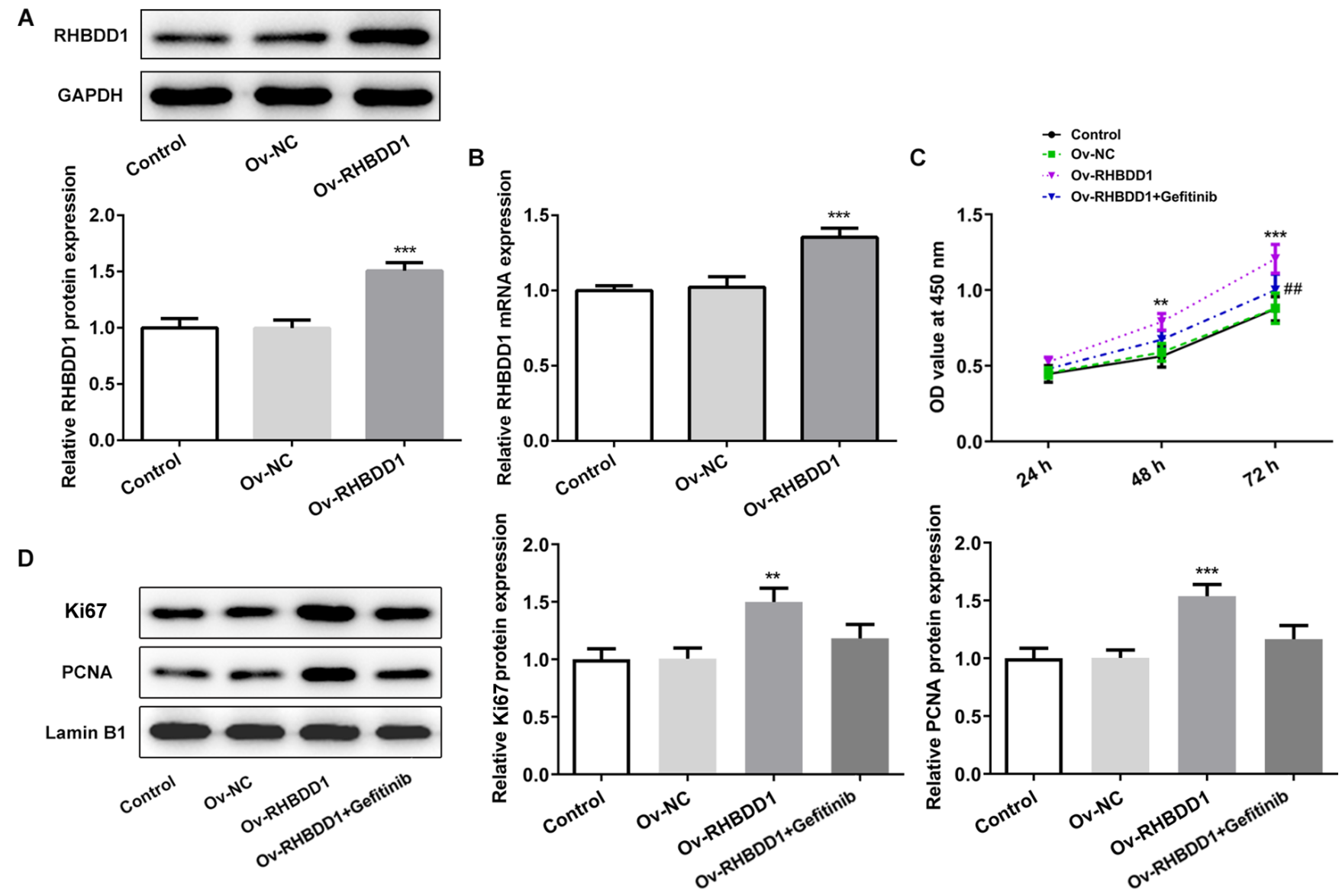

Figure 5. RHBDD1 promotes cell proliferation via EGFR/AKT signaling pathway in renal cell carcinoma. (A and B) RHBDD1 protein and mRNA expression levels in 7860 cells were analyzed using western blotting and reverse transcription-quantitative PCR, respectively. (C) Cell proliferation was analyzed using a Cell Counting Kit-8 assay. (D) Western blotting was used to analyze Ki67 and PCNA expression levels. ${ }^{* *} \mathrm{P}<0.01,{ }^{* * *} \mathrm{P}<0.001$ vs. Ov-NC; ${ }^{\# /} \mathrm{P}<0.01$ vs. Ov-RHBDD1. RHBDD1, rhomboid domain-containing protein 1; PCNA, proliferating cell nuclear antigen; Ov, overexpression; NC, negative control; OD, optical density.

transfected into the 7860 cell line. Data from western blotting (Fig. 5A) and RT-qPCR (Fig. 5B) analyses both demonstrated that transfection with Ov-RHBDD1 significantly upregulated RHBDD1 expression levels compared with the Ov-NC group. The results of CCK- 8 assay revealed that the promoting effect of RHBDD1 on cell proliferation was markedly reversed following treatment with gefitinib, an EGFR inhibitor (Fig. 5C). Similarly, the upregulation of proliferation-associated proteins, including Ki67 and PCNA was partly reversed by gefitinib treatment (Fig. 5D). These results suggested that RHBDD1 may promote cell proliferation via EGFR/AKT signaling pathway in RCC. 

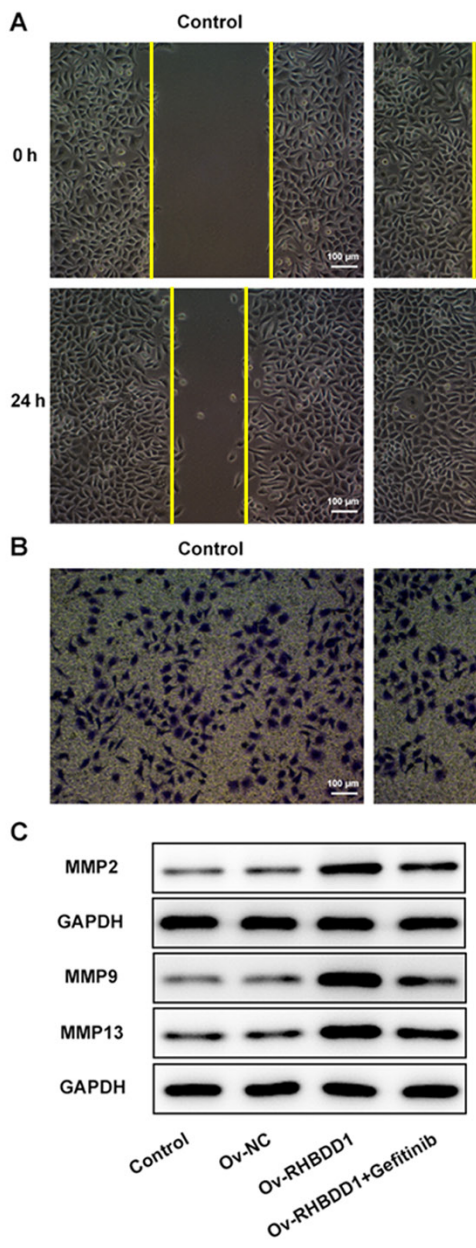

D

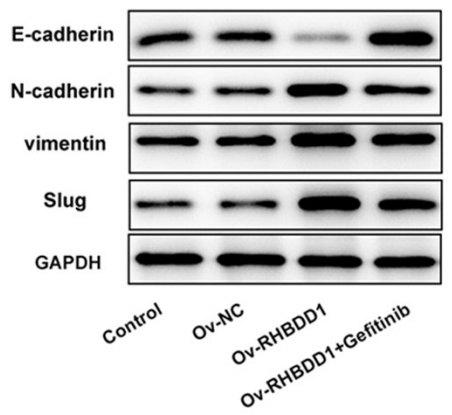

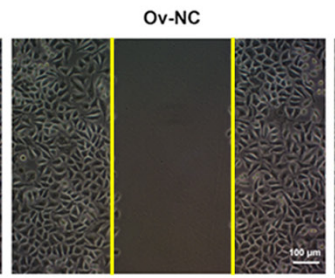

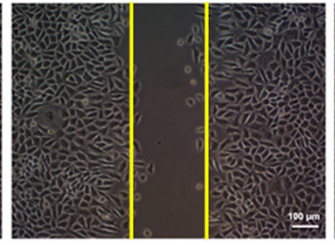

Ov-RHBDD1

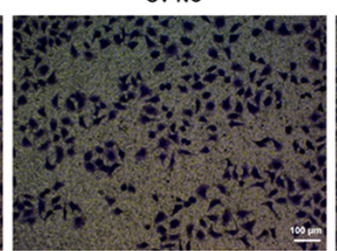

Ov-RHBDD1
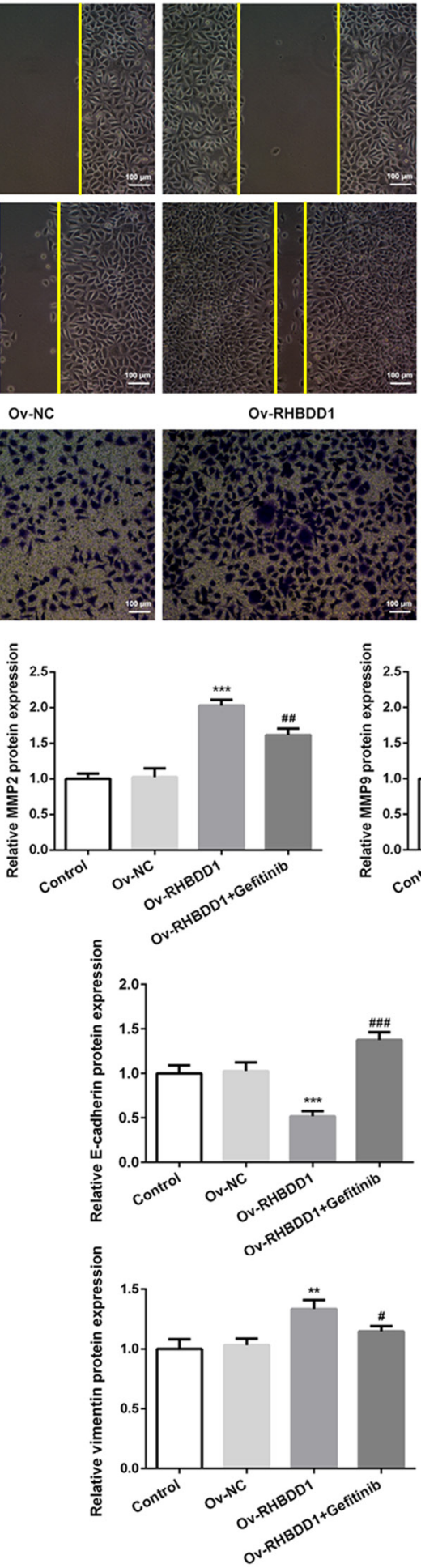

Ov-RHBDD1+Gefitinib

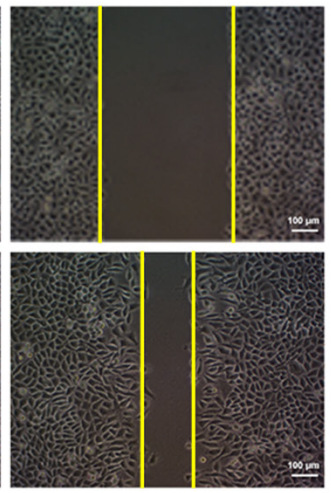

Ov-RHBDD1+Gefitinib
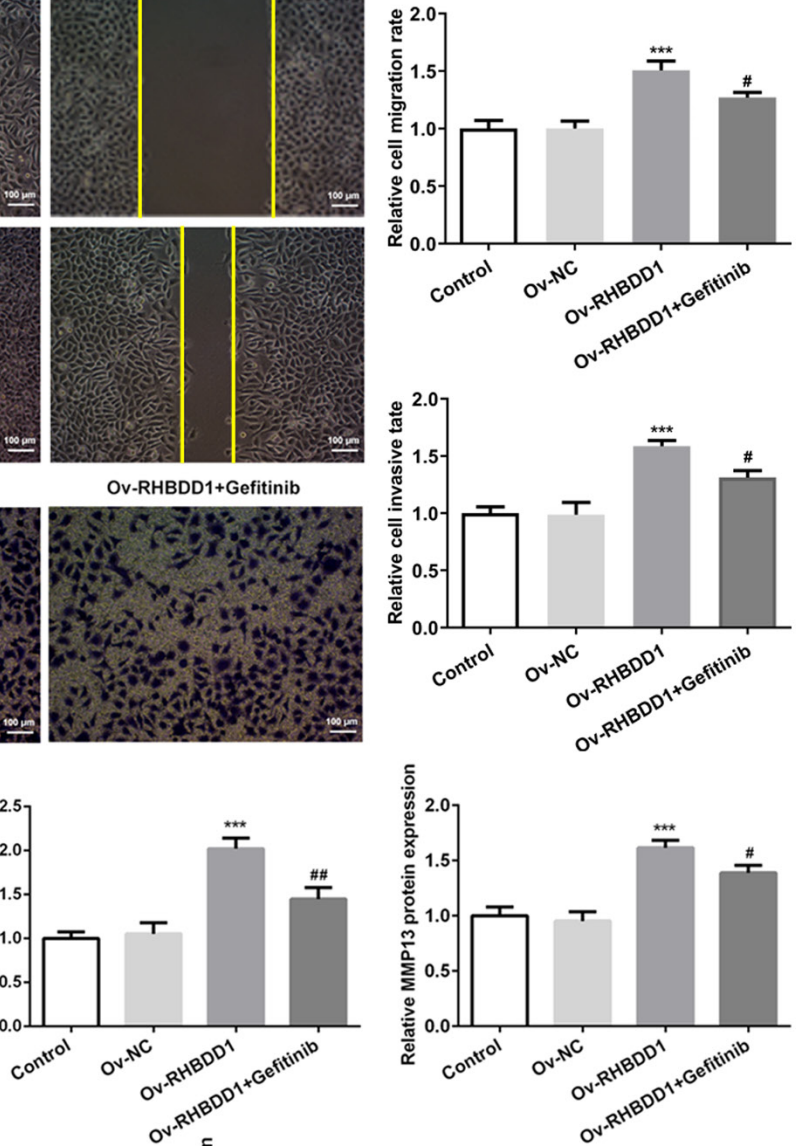

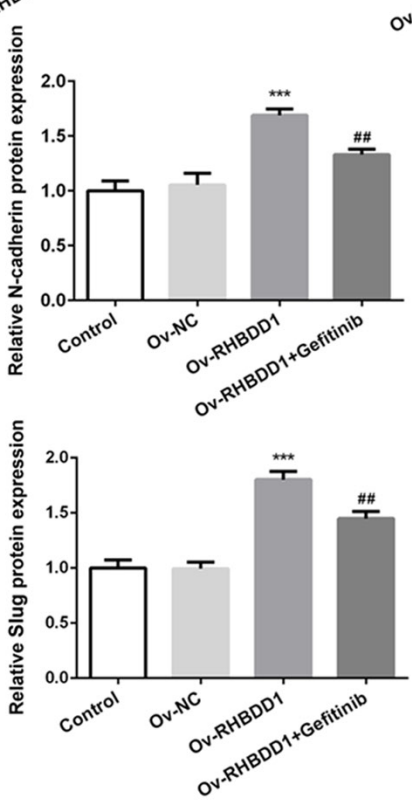

Figure 6. RHBDD1 promotes cell migration, invasion and EMT via EGFR/AKT signaling pathway in renal cell carcinoma. (A) Wound healing assay was performed to determine cell migration (magnification, x100; scale bar, $100 \mu \mathrm{m}$ ). (B) Transwell assay was performed to determine cell invasion (magnification, x100; scale bar, $100 \mu \mathrm{m}$ ). Western blotting was performed to analyze the expression levels of key proteins involved in (C) cell migration and invasion, such as MMP2, MMP9 and MMP13 and (D) EMT, such as E-cadherin, N-cadherin, vimentin and Slug. ${ }^{* *} \mathrm{P}<0.01,{ }^{* * *} \mathrm{P}<0.001$ vs. Ov-NC; ${ }^{\#} \mathrm{P}<0.05,{ }^{\# \#} \mathrm{P}<0.01$,

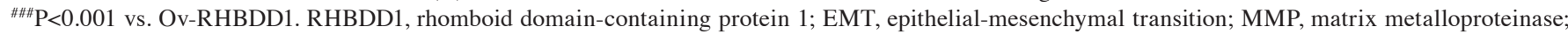
Ov, overexpression; NC, negative control.

RHBDD1 promotes cell migration, invasion and EMT via EGFR/AKT signaling pathway in RCC. The underlying mechanism through which RHBDD1 may promote RCC cell invasion and migration was also identified. Transfection with
Ov-RHBDD1 significantly promoted cell migration compared with the Ov-NC group, while this effect was significantly abolished by gefitinib treatment (Fig. 6A). Similarly, RHBDD1 overexpression significantly promoted cell invasion compared 
A

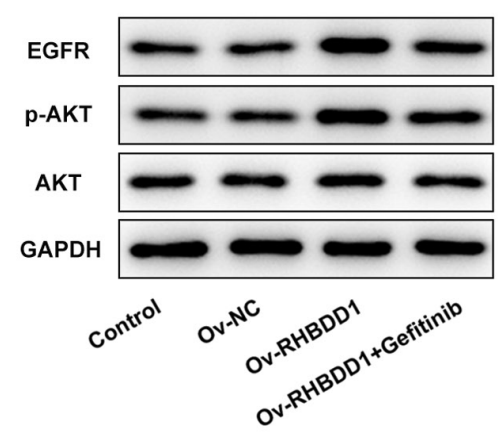

B

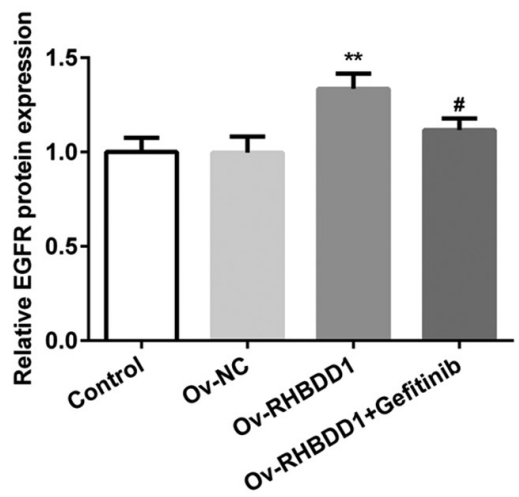

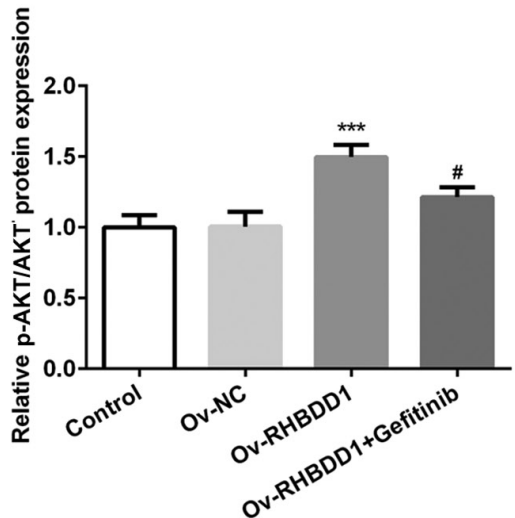

Figure 7. Gefitinib effectively blocks EGFR/AKT signaling pathway in renal cell carcinoma. (A) Western blotting was used to analyze the expression levels of EGFR, p-AKT and AKT. (B) Expression levels of EGFR, p-AKT and AKT from part (A) were semi-quantified. ${ }^{* *} \mathrm{P}<0.01,{ }^{* * *} \mathrm{P}<0.001$ vs. Ov-NC; ${ }^{*} \mathrm{P}<0.05$ vs. Ov-RHBDD1. RHBDD1, rhomboid domain-containing protein 1; p-, phosphorylated; Ov, overexpression; NC, negative control.

with the Ov-NC group and this effect was also rescued by gefitinib treatment (Fig. 6B). In addition, the expression levels of key proteins related to cell migration and invasion (MMP2, MMP9 and MMP13) were significantly upregulated following transfection with Ov-RHBDD compared with the Ov-NC group, while gefitinib treatment downregulated the protein expression levels of MMP2, MMP9 and MMP13 (Fig. 6C). Simultaneously, the expression levels of proteins involved in EMT were also evaluated. Gefitinib treatment reversed the downregulation in E-cadherin expression levels and upregulation in N-cadherin, vimentin and Slug expression levels induced by RHBDD1 overexpression (Fig. 6D). These results suggested that RHBDD1 may promote cell migration, invasion and EMT via EGFR/AKT signaling pathway in RCC.

Gefitinib effectively inhibits EGFR/AKT signaling pathway in $R C C$. The expression levels of proteins involved in EGFR/AKT signaling pathway (EGFR and p-AKT) were significantly upregulated following RHBDD1 overexpression compared with the Ov-NC group, while the expression levels of EGFR and p-AKT were downregulated following gefitinib treatment (Fig. 7A and B). Collectively, these results suggested that gefitinib may effectively suppress EGFR/AKT signaling pathway in RCC.

\section{Discussion}

RCC is highly resistant to radiotherapy and chemotherapy, and immunotherapy is currently less effective in treating the disease $(4,5)$. In recent years, with the continuous development of medical technology and increase in research on the pathogenesis of RCC, molecular targeted drugs (sunitinib, pazopanib, axitinib and everolimus) have provided a novel method for the treatment of advanced RCC $(25,26)$. The present study aimed to determine the biological characteristics of RHBDD1 and analyzed the potential value of RHBDD1 as a therapeutic target for RCC.

Targeted anticancer therapy has been widely applied for the treatment of malignant cancers following surgical resection, radiotherapy and chemotherapy (25). A previous study reported that silencing of RHBDD1 can significantly suppress cell proliferation and promote the apoptosis of HepG2 cells (27). Accumulating studies have begun to pay attention to the role of MMPs and EMT in cancer metastasis $(7,10)$. Zhao et al (21) verified that downregulation of RHBDD1 can inhibit the migratory and invasive abilities and EMT of breast cancer cells. Moreover, Huanget al (22) demonstrated that RHBDD1 knockdown can inhibit breast cancer cell migration and invasion by repressing MMP2/9 protein levels and EMT. In addition, it has been confirmed that RHBDD1 expression in colorectal tumor tissues is positively associated with distal metastasis and lymphatic metastasis (28). The results of the present study revealed that RHBDD1 expression levels were markedly upregulated in human RCC cell lines. The overexpression of RHBDD1 could significantly promote cell proliferation, migration, invasion and EMT in RCC, while the application of EGFR inhibitor (gefitinib) reversed the effects of RHBDD1 overexpression.

EGFR, a member of the family of protein tyrosine kinase receptors, plays a crucial role in the proliferation, apoptosis, invasive ability and metastasis of tumor cells $(29,30)$. EGFR inhibitors are vital antitumor agents used to prevent the progression of multiple types of cancer $(14,15)$. A previous study demonstrated that promoting EGFR degradation and suppressing EGF-induced AKT signaling can inhibit the growth and metastasis of kidney clear cell carcinoma (31). Moreover, RHBDD1 can activate EGFR signaling pathway in colorectal cancer (23). As expected, the present results revealed that knockdown of RHBDD1 suppressed EGFR/AKT signaling pathway activity in RCC.

In conclusion, the findings of the present study demonstrated that RHBDD1 promoted cell proliferation, migration, invasion and EMT through modulating EGFR/AKT signaling pathway in RCC. The current data highlighted a pivotal role of RHBDD1 in the tumorigenesis of RCC, which may provide an improved understanding of the pathogenesis of RCC and a novel therapeutic target or biomarker for RCC.

\section{Acknowledgements}

Not applicable.

\section{Funding}

No funding was received. 


\section{Availability of data and materials}

The datasets used and/or analyzed during the present study are available from the corresponding author on reasonable request.

\section{Authors' contributions}

ML, LC, XW, CL and CZ designed the present study. ML, LC, XW, YY, WJ, GB, ZG, JG and JZ performed the experiments. ML, LC, XW, YY, WJ and GB analyzed and interpreted the data. ML, LC, XW, YY, WJ, GB, CL and CZ drafted the manuscript. CL and CZ revised the manuscript. All authors have read and approved the final manuscript. CZ and CL confirm the authenticity of all the raw data.

\section{Ethics approval and consent to participate}

Not applicable.

\section{Patient consent for publication}

Not applicable.

\section{Competing interests}

The authors declare that they have no competing interests.

\section{References}

1. Chen S, Wang Y, Xiong Y, Peng T, Lu M, Zhang L and Guo Z: Wild-type IDH1 inhibits the tumor growth through degrading HIF- $\alpha$ in renal cell carcinoma. Int J Biol Sci 17: 1250-1262, 2021.

2. Huang T, Wang X, Yang X, Ji J, Wang Q, Yue X and Dong Z: long non-coding RNA DUXAP8 enhances renal cell carcinoma progression via downregulating miR-126. Med Sci Monit 24: 7340-7347, 2018.

3. Cohen HT and McGovern FJ: Renal-cell carcinoma. N Engl J Med 353: 2477-2490, 2005.

4. Zhu H, Wang Z, Xu Q, Zhang Y, Zhai Y, Bai J, Liu M, Hui Z and $\mathrm{Xu}$ N: Inhibition of STAT1 sensitizes renal cell carcinoma cells to radiotherapy and chemotherapy. Cancer Biol Ther 13: 401-407, 2012.

5. Teng ZY, Cheng XL, Cai XT, Yang Y, Sun XY, Xu JD, Lu WG, Chen J, Hu CP, Zhou Q, et al: Ancient Chinese Formula Qiong-Yu-Gao Protects Against Cisplatin-Induced Nephrotoxicity Without Reducing Anti-tumor Activity. Sci Rep 5: 15592, 2015.

6. Flippot R, Escudier B and Albiges L: Immune Checkpoint Inhibitors: Toward New Paradigms in Renal Cell Carcinoma. Drugs 78: 1443-1457, 2018.

7. Zhong Y, Lu YT, Sun Y, Shi ZH, Li NG, Tang YP and Duan JA: Recent opportunities in matrix metalloproteinase inhibitor drug design for cancer. Expert Opin Drug Discov 13: 75-87, 2018.

8. Liu QH, Wang Y, Yong HM, Hou PF, Pan J, Bai J and Zheng JN: XRCC1 serves as a potential prognostic indicator for clear cell renal cell carcinoma and inhibits its invasion and metastasis through suppressing MMP-2 and MMP-9. Oncotarget 8: 109382-109392, 2017.

9. Liang F, Wang YG and Wang C: Metformin inhibited growth, invasion and metastasis of esophageal squamous Cell Carcinoma in vitro and in vivo. Cell Physiol Biochem 51: 1276-1286, 2018.

10. Tsai JH, Donaher JL, Murphy DA, Chau S and Yang J: Spatiotemporal regulation of epithelial-mesenchymal transition is essential for squamous cell carcinoma metastasis. Cancer Cell 22: 725-736, 2012.

11. Zhang P, Sun Y and Ma L: ZEB1: At the crossroads of epithelial-mesenchymal transition, metastasis and therapy resistance. Cell Cycle 14: 481-487, 2015.

12. Pan G, Liu Y, Shang L, Zhou F and Yang S: EMT-associated microRNAs and their roles in cancer stemness and drug resistance. Cancer Commun (Lond) 41: 199-217, 2021.
13. Chuang MJ, Sun KH, Tang SJ, Deng MW, Wu YH, Sung JS, Cha TL and Sun GH: Tumor-derived tumor necrosis factor-alpha promotes progression and epithelial-mesenchymal transition in renal cell carcinoma cells. Cancer Sci 99: 905-913, 2008.

14. Liao BC, Lin CC and Yang JC: Second and third-generation epidermal growth factor receptor tyrosine kinase inhibitors in advanced nonsmall cell lung cancer. Curr Opin Oncol 27: 94-101, 2015.

15. Singh D, Attri BK, Gill RK and Bariwal J: Review on EGFR Inhibitors: Critical Updates. Mini Rev Med Chem 16: 1134-1166, 2016.

16. Wang J, Xu H, Cheng X, Yang J, YanZ, Ma H,Zhao Y, Ommati MM, Manthari RK and Wang J: Calcium relieves fluoride-induced bone damage through the PI3K/AKT pathway. Food Funct 11: $1155-1164,2020$.

17. Manning BD and Toker A: AKT/PKB Signaling: Navigating the Network. Cell 169: 381-405, 2017.

18. Brown JS and Banerji U: Maximising the potential of AKT inhibitors as anti-cancer treatments. Pharmacol Ther 172: 101-115, 2017.

19. Liu L, Miao L, Liu Y, Qi A, Xie P, Chen J and Zhu H: S100A11 regulates renal carcinoma cell proliferation, invasion, and migration via the EGFR/Akt signaling pathway and E-cadherin. Tumour Biol 39: 1010428317705337, 2017.

20. Wang Y, Guan X, Fok KL, Li S,Zhang X, Miao S, Zong S, Koide SS, Chan HC and Wang L: A novel member of the Rhomboid family, RHBDD1, regulates BIK-mediated apoptosis. Cell Mol Life Sci 65: 3822-3829, 2008

21. Zhao C, Ling X, Li X, Hou X and Zhao D: MicroRNA-138-5p inhibits cell migration, invasion and EMT in breast cancer by directly targeting RHBDD1. Breast Cancer 26: 817-825, 2019.

22. Huang C, Ji X, Peng Y, Wu M, Wu W, Luo Y, Cheng G and Zhu Y: Silencing of rhomboid domain containing 1 to inhibit the metastasis of human breast cancer cells in vitro. Iran J Basic Med Sci 21: 1161-1166, 2018.

23. Song W, Liu W, Zhao H, Li S, Guan X, Ying J, Zhang Y, Miao F, Zhang M, Ren X, et al: Rhomboid domain containing 1 promotes colorectal cancer growth through activation of the EGFR signalling pathway. Nat Commun 6: 8022, 2015.

24. Livak KJ and Schmittgen TD: Analysis of relative gene expression data using real-time quantitative PCR and the 2(-Delta Delta C(T)) Method. Methods 25: 402-408, 2001.

25. Kitano H, Kitadai Y, Teishima J, Yuge R, Shinmei S, Goto K, Inoue S, Hayashi T, Sentani K, Yasui W, et al: Combination therapy using molecular-targeted drugs modulates tumor microenvironment and impairs tumor growth in renal cell carcinoma. Cancer Med 6: 2308-2320, 2017.

26. Hawkins R, Fife K, Hurst M, Wang M, Naicker N, Nolasco S, Eisen T, Matakidou A and Gordon J: Treatment patterns and health outcomes in metastatic renal cell carcinoma patients treated with targeted systemic therapies in the UK. BMC Cancer 20: 670, 2020.

27. Liu XN, Tang ZH, Zhang Y, Pan QC, Chen XH, Yu YS and Zang GQ: Lentivirus-mediated silencing of rhomboid domain containing 1 suppresses tumor growth and induces apoptosis in hepatoma HepG2 cells. Asian Pac J Cancer Prev 14: 5-9, 2013.

28. Zhang M, Miao F, Huang R, Liu W, Zhao Y, Jiao T, Lu Y, Wu F, Wang X, Wang H, et al: RHBDD1 promotes colorectal cancer metastasis through the Wnt signaling pathway and its downstream target ZEB1. J Exp Clin Cancer Res 37: 22, 2018.

29. Souza JL, Martins-Cardoso K, Guimarães IS, de Melo AC, Lopes AH, Monteiro RQ and Almeida VH: Interplay Between EGFR and the Platelet-Activating Factor/PAF Receptor Signaling Axis Mediates Aggressive Behavior of Cervical Cancer. Front Oncol 10: 557280, 2020.

30. Zhou Q, Huang T, Jiang Z, Ge C, Chen X, Zhang L, Zhao F, Zhu M, Chen T, Cui Y, et al: Upregulation of SNX5 predicts poor prognosis and promotes hepatocellular carcinoma progression by modulating the EGFR-ERK1/2 signaling pathway. Oncogene 39: 2140-2155, 2020.

31. Yin L, Gao S, Shi H, Wang K, Yang H and Peng B: TIP-B1 promotes kidney clear cell carcinoma growth and metastasis via EGFR/AKT signaling. Aging (Albany NY) 11: 7914-7937, 2019.

This work is licensed under a Creative Commons Attribution-NonCommercial-NoDerivatives 4.0 International (CC BY-NC-ND 4.0) License. 